\title{
"Myope comme une taupe, muette comme une carpe, mais... malin comme un singe ". Les animaux anthropomorphisés porteurs de handicaps dans les albums jeunesse
}

\author{
Lemoine Lise $^{\text {a }}$
}

\section{Mietkiewicz Marie-Claude ${ }^{b}$}

\section{Schneider Benoît ${ }^{\mathrm{c}}$}

\author{
a Laboratoire de psychologie, cognition, comportement, communication (LP3C), Université de Rennes 2, France. \\ ${ }^{\mathrm{b}}$ Laboratoire Interpsy, Université de Lorraine, France. \\ ${ }^{\mathrm{c}}$ Laboratoire lorrain de psychologie et neurosciences de la dynamique des comportements (2LPN) Université de \\ Lorraine, France.
}

Correspondance : Lise Lemoine, Université Rennes 2, Place du recteur Henri Le Moal, CS 24307 / 35043 Rennes cedex, France.

Courriel : lise.lemoine@univ-rennes2.fr

Texte reçu le 11 avril 2018 et accepté le 16 juillet 2019

http://www.bulletindepsychologie.net

Résumé : Les ouvrages de jeunesse sur le thème du handicap peuvent être un support pour accompagner les situations pédagogiques, en particulier dans le cadre de politiques inclusives. Vingt-huit albums destinés à des enfants âgés de trois à cinq ans, parus entre 2000 et 2016 et mettant en scène des animaux avec handicap ont été recensés. L'étude porte sur les caractéristiques de l'anthropomorphisation, les relations entre héros avec handicap et réseau familial et social, et les «messages » portés par l'histoire. Les degrés de

l'anthropomorphisation sont divers et complexes et on ne peut présupposer d'effets simples et linéaires. L'étude des travaux portant sur les liens entre référence à l'animal et incitation aux comportements prosociaux chez l'enfant invite à un examen attentif des contextes éducatifs dans lesquels ces ouvrages peuvent servir de support.

\section{"Blind as a Bat, Lame as a Duck, but... Smart as a Monkey". Anthropomorphized Animals with} Disabilities in Children's Picture Books

Abstract: Children's books about disability can be used as a help in pedagogical situations, particularly as part of inclusive policies. We have identified 28 picture books for 3- to 5-year-old children, released between 2000 and 2016, which represent animals with disabilities, and examined the main features of anthropomorphization, the relationships between the disabled protagonist and their social and family network, and the "messages" conveyed by the narrative. The types of representation and the extent of anthropomorphization are varied and complex, so simple and linear effects cannot be expected. The examination of the works available on the connection between references to animals and encouragement of pro-social behaviours in children suggests that a careful examination of the educational contexts in which such books can be used is needed. 


\section{INTRODUCTION}

Lorsqu'on s'intéresse à la représentation du handicap dans la littérature jeunesse, qu'il s'agisse de troubles du développement, de déficiences sensorielles ou motrices (Joselin, 2010, 2013 ; Lemoine, Mietkiewicz, Schneider, 2012, 2016a, 2016b, 2017), on est surpris de constater la place qu'occupent les animaux anthropomorphes dans les albums jeunesse. Merlette avec autisme, lapin porteur de trisomie 21, souriceaux atteints de cécité, fourmi dont une patte est atrophiée... un étrange bestiaire peuple les livres à destination des jeunes enfants à qui l'on raconte des histoires illustrées. Nous avons porté notre intérêt sur ces albums particuliers dans lesquels les problématiques liées au handicap sont abordées par le truchement des représentations animales afin d'en saisir les caractéristiques et de tenter d'en approcher l'intérêt.

Cet intérêt se justifie d'autant qu'il a des implications pédagogiques telles qu'attestées par les programmes de l'école maternelle puisque celle-ci « assure ainsi une première acquisition des principes de la vie en société ». L'accueil et la scolarisation des enfants handicapés participent à cet enjeu pour ces enfants eux-mêmes et contribuent à développer pour tous un regard positif sur les « différences » en invitant notamment les enseignants à « favoriser les interactions entre enfants et créer les conditions d'une attention partagée, la prise en compte du point de vue de l'autre ». En effet, « à travers les situations concrètes de la vie de la classe, une première sensibilité aux expériences morales (sentiment d'empathie, expression du juste et de l'injuste, questionnement des stéréotypes...) se construit. Les histoires lues, contes et saynètes y contribuent ; la mise en scène de personnages fictifs suscite des possibilités diversifiées d'identification et assure en même temps une mise à distance suffisante. » (BO spécial n ${ }^{\circ} 1 \mathrm{du} 26$ mars 2015) ${ }^{1}$. Les ouvrages destinés aux très jeunes enfants peuvent donc 
contribuer à leur développement, ce que soulève d'ailleurs Virginie Meyer sur le site Gallica ${ }^{2}$ en mentionnant que ce sont dans les années 1980-1990 que ces albums se sont développés après la mise en évidence de leur bienfait par les pédopsychiatres et psychanalystes.

Après avoir rapidement dressé un panorama des différentes places qu'occupent les animaux dans la littérature jeunesse, nous définirons les critères d'inclusion dans le corpus d'étude. L'analyse thématique des ouvrages sélectionnés permettra ensuite d'identifier les particularités des animaux mis en scène et les caractéristiques des trames narratives. La discussion abordera l'intérêt et discutera la spécificité de l'usage de l'animal anthropomorphe dans l'approche du handicap.

\section{LES ANIMAUX DE LA LITTÉRATURE JEUNESSE}

Chiens, chats, ours, loups, domestiques ou sauvages, les animaux occupent une place importante dans la littérature jeunesse et tout particulièrement dans les albums destinés aux tout-petits. On les trouve dans les innombrables imagiers qui permettent aux bambins de reconnaître les espèces et d'apprendre à les nommer, et dans des albums documentaires qui les présentent dans leur milieu naturel comme, par exemple, la série Roman des bêtes (Panache l'écureuil, Quipic le hérisson, Froux le lièvre...) publiée à l'initiative de Paul Foucher, sous le label Père Castor dès les années 1930 et régulièrement rééditée depuis. On trouve aussi des animaux en position de compagnon de l'enfant-héros : confidents fidèles ou protagonistes actifs, ils jouent un rôle effectif et apportent leur contribution au dénouement de l'histoire (par exemple, Le chien bleu de Nadja). Enfin, dans cette classification sommaire, nous distinguerons les animaux anthropomorphes, des animaux qui se comportent comme des

2. http://gallica.bnf.fr/blog/29072016/heros-de-la-litterature-pour-la-jeunesse-episode-5-doudous-et-braves-betes [consulté le 07.03.2018] 
humains, dans des récits qui racontent les déboires, les joies, les peurs et les exploits de petits héros : pingouin (Tchoupi), âne (Trotro), ours (Petit Ours Brun).

La représentation anthropomorphe des animaux est un dispositif littéraire très ancien puisque, déjà au VII ${ }^{\mathrm{e}}$ siècle avant Jésus-Christ, les fables attribuées à Ésope mettent en scène des animaux auxquels sont prêtés des caractéristiques et des comportements humains. Quelques siècles plus tard, Phèdre ( $\mathrm{I}^{\mathrm{er}}$ siècle) écrit des récits courts dans lesquels les animaux proposent de façon plaisante une leçon de vie et de morale. Le roman de Renart ( $\mathrm{XI}^{\mathrm{e}}$ siècle), écrit en vers et raconté par les trouvères, est un ensemble médiéval de récits animaliers dans lequel le monde des animaux est un miroir et une critique du monde féodal. Chaque chapitre de ce poème allégorique, à travers les aventures de deux personnages principaux, Renart, le goupil espiègle et rusé, et Ysengrin, le loup stupide et cruel et de nombreux autres animaux dans des rôles secondaires, dénonce l'hypocrisie des chevaliers, des hommes d'église, des courtisans et des seigneurs. Au XVII ${ }^{\mathrm{e}}$ siècle, Les Fables de Jean de la Fontaine s'inscrivent dans cette tradition de dénonciation des travers des hommes et de la société en déjouant la censure des puissants.

Sans multiplier les exemples, d'autant que nous allons nous intéresser aux livres pour les très jeunes enfants, mentionnons encore deux œuvres remarquables plus récentes dont les héros animaux donnent à réfléchir quant aux les dérives où peuvent tomber les sociétés humaines. La ferme des animaux de Georges Orwell (1945), satire de la révolution russe et du stalinisme, est le récit d'une dystopie dans laquelle la révolte des animaux justifiée par leur espoir de vivre autonomes, égaux et solidaires dans la paix, génère des comportements contraires à toutes les valeurs initialement prônées et conduit à un régime totalitaire. Rhinocéros, d'Eugène Ionesco (1959), est une métaphore de la montée du nazisme et du fascisme ; petit à petit, les habitants de la ville se transforment en rhinocéros, mammifères dont la couleur rappelle celle des uniformes des SS. 
Ainsi, force est de constater que l'animal anthropomorphe est inscrit dans l'histoire et dans la tradition littéraire au fil des siècles, mais son apparition n'est mentionnée dans la littérature jeunesse qu'au début du XX⿳亠丷厂犬 siècle (Diament, 2008 ; Poslaniec, 2008 ; Nièvre-Chevrel, 2009), d'abord en Angleterre avec Peter Rabbit de Beatrix Potter (1902) et, quelques décennies plus tard, en France avec l'éléphant Babar de Jean de Brunhoff (1931). Depuis, les animaux sont à foison dans les albums où ils vivent des aventures variées, affrontent des difficultés empruntées au monde des humains et inventent des solutions pour se sortir de tous les mauvais pas. La tradition d'anthropomorphisme est donc bien installée puisqu'une étude (Marriott, 2002) ayant passé mille albums jeunesse au crible a montré que plus de la moitié mettaient en scène des animaux, dont $2 \%$ à peine étaient crédités de comportements réalistes. Savier (1988) explicite l'intérêt de ce recours à l'animal : « Les plus jeunes ont une sympathie, favorisée par les adultes, pour les animaux. Dans les livres, déguisés en humains, animaux prétextes, animaux modèles, asexués, ils se métamorphosent en petits êtres demandant protection et éducation. Ils semblent refléter la situation enfantine moyenne et, gommant les distinctions sociales, raciales ou culturelles, ils facilitent, dans la sécurité de l'intemporalité, l'identification. » (p. 68), alors que les fables mettent l'accent sur ces dimensions. Léon (2007) souligne la particularité de ces albums, qui favorisent d'autant plus l'identification qu'ils mettent à distance : « Dans les collections de premiers albums, presque tous les éditeurs proposent des ouvrages qui présentent des animaux très humanisés (...). Ces livres “jouent à fond” sur l'effet de miroir qui permet, paradoxalement, de prendre du recul par rapport à soi-même. » (p. 57). Perrin (2001) fait l'hypothèse selon laquelle les albums anthropomorphiques permettent une plus grande distanciation des enfants par rapport au sujet, et sont donc propices à des discussions plus riches, en prenant l'exemple de la nouvelle Le chat de Tigali (Daeninckx, 2007), journal intime fictif qui évoque un crime raciste perpétré à l'encontre d'un chat : « Le choix du chat comme victime permet à l'auteur d'aller le plus loin 
possible dans le récit du racisme, jusqu'à l'inacceptable dans une œuvre pour enfant. » (p. 32). Ce contre-exemple, puisque la question du racisme, loin d'être escamotée, est centrale, atteste d'une évolution récente de la littérature jeunesse qui s'empare depuis quelques décennies des sujets de société comme, pour ne donner que quelques exemples, le mariage pour tous ( $C$ 'est la vie mon poussin, Gouichoux, 2015), l'homoparentalité (Mes deux papas, Parachini-Deny et Beal, 2015) et des sujets difficiles, dont la mort (Maman ourse est partie, Gouichoux, 2003).

Animaux anthropomorphes plutôt qu'humains zoomorphes, selon Nièvre-Chevrel (2009), ils sont le plus souvent des animaux-enfants qui vivent en famille avec parents, frères et sœurs et grands-parents, se comportent dans la vie quotidienne comme des enfants : ils mangent à table, dorment dans des lits, vont à l'école, fêtent leurs anniversaires et n'ont, au final, d'animal que l'apparence. Girafe ou hibou, grenouille ou tigre, kangourou ou loup, ils parlent et manifestent leurs émotions dans une large gamme d'expressions (colère, tristesse, joie...). Bourre (2017, citée par Armengaud, 2017, p. 187) auteure et illustratrice reconnaît : « Nous avons pris en otage tout le monde animal pour enseigner à nos petits comment bien grandir, en leur faisant un peu peur, en les faisant rêver, en les mettant en garde, en les amusant. »

Le recours à l'animal se signale aussi dans les tests projectifs pour jeunes enfants invités à se raconter à travers les aventures d'un petit cochon («Patte Noire» de Louis Corman, 1972) des fables de Louisa Duss (1950) ou du test des Contes de Jacqueline Royer (1990). Faisant pendant aux animaux pris pour des humains dans les livres, Gravillon (2017) souligne la fréquence avec laquelle les surnoms que donnent les parents à leurs jeunes enfants empruntent au bestiaire animal : dans l'intimité familiale, la relation d'affection donne lieu à des « mon petit loup », « ma puce », « mon lapin », etc., autant de surnoms animaliers pour dire tendresse et protection que renforcent les adjectifs possessifs qui les accompagnent. 
Mais notre intention n'est pas de décliner toutes les facettes de la relation entre l'enfant et l'animal ni d'explorer la place de l'animal dans l'imaginaire ou dans la réalité quand il devient un auxiliaire des thérapeutes et éducateurs d'enfant en situation de handicap. Plus modestement et de façon très circonscrite, nous proposons d'analyser les caractéristiques des albums pour les tout jeunes enfants lorsqu'ils mettent en scène des animaux anthropomorphes qui présentent des caractéristiques physiques, mentales ou psychiques faisant entrave à leur développement. Nous tenterons d'examiner dans quelle mesure ces albums mis à disposition des jeunes enfants peuvent représenter des supports susceptibles de les aider à développer des tendances à l'empathie et à acquérir des attitudes de tolérance face aux différences.

\section{LES ANIMAUX EN SITUATION DE HANDICAP : MÉTHODE ET QUESTIONS DE RECHERCHE}

Pour constituer le corpus d'étude, nous avons sélectionné, parmi les très nombreux livres publiés (ou réédités) entre le $1^{\mathrm{er}}$ janvier 2000 et le 31 décembre 2016 qui mettent en scène des animaux avec handicap ou déficience, les albums à destination des enfants de 3-5 ans (classes maternelles). Pour repérer les ouvrages, nous avons consulté les sites web dédiés à la littérature jeunesse, proposés par des associations ou les réseaux CANOPE (réseaux de création et d'accompagnement pédagogiques), et les informations recueillies auprès des bibliothécaires et libraires spécialisés. Nous avons d'abord considéré comme critère minimal d'anthropomorphisme la présence, entre les protagonistes du récit, d'échanges verbaux dans la langue humaine et non par des cris spécifiques des différentes espèces. Nous avons ensuite retenu les albums dans lesquels l'animal peut être considéré comme porteur d'un handicap en référence à la définition de la législation française : « Constitue un handicap, au sens de la présente loi, toute limitation d'activité ou de restriction de participation à la vie en société subie dans son environnement par une personne en raison d'une altération substantielle, 
durable ou définitive d'une ou plusieurs fonctions physiques, sensorielles, mentales, cognitives ou psychiques, d'un polyhandicap ou d'un trouble de santé invalidant. ${ }^{3}$ Enfin, nous avons sélectionné les livres dans lesquels l'animal anthropomorphisé est un enfantanimal de façon à centrer notre analyse sur les entraves au développement induites par le handicap dont l'animal est atteint.

Au final, cette recherche porte sur vingt-huit titres dont la liste figure en annexe. Dans la suite du propos, chaque ouvrage sera référencié par son numéro d'ordre dans cette liste.

Relativement à la date de publication, il y a cinq ouvrages dont la dernière édition se situe entre 2000 et 2004 [3, 4, 22, 18, 22], huit entre 2005 et $2010[1,2,7,15,16,20,25,27]$ et treize entre 2011 et $2016[6,8,10,11,12,13,14,17,19,21,23,24,26]$. Deux ouvrages ont été réédités en 2017 [5,9]. Cinq ouvrages ont traversé le temps en ayant été réédités à plusieurs reprises : depuis 1963 : [4] $(1963,1966,1976,2002) ; 1971$ : [18] $(1971,1972$, $1973,2001) ; 1984:[3](1984,1986,2002) ; 1990:$ [5] $(1990,2000,2002,2017) ; 1991$ : [9] (1991, 1993, 1999, 2003, 2017).

Quant à l'édition d'origine, vingt ouvrages ont été publiés d'emblée en France [1, 2, 3, 5, 6, 8, 9, 10, 11 (île de la Réunion), 12, 13, 14, 15, 16, 19, 20, 21, 22, 23, 26] ; huit ouvrages ont été publiés initialement en Angleterre [4], en Belgique [7, 24], en Bulgarie [17], aux ÉtatsUnis [18, 28], en Allemagne [25] et en Autriche [27].

Les modalités éditoriales apparaissent hétérogènes : grandes maisons d'édition, éditeurs très locaux, albums mis en ligne par des structures hospitalières ; auteurs « professionnels » ayant publié de nombreux ouvrages portant sur divers thèmes ou professionnels du champ médico-social à l'origine d'un projet éditorial spécifique...

3. Article 2 de la loi $n^{\circ} 2005-102$ pour l'égalité des droits et des chances, la participation et la citoyenneté des personnes handicapées. 
Pour rendre compte de la richesse du matériel tout en adoptant une méthode la plus objective possible, nous avons procédé à une analyse de contenu thématique en proposant une grille permettant de décrire les caractéristiques essentielles des héros, les modalités de l'anthropomorphisation, les rapports des enfants-héros avec leur environnement familial et social et la trame narrative au regard des difficultés rencontrées. Nous aborderons donc successivement les points suivants :

— les supports de l'anthropomorphisation : nous repérerons les types d'animaux et de handicaps dont ils sont porteurs ; puis nous évaluerons la nature de l'anthropomorphisation en proposant d'en évaluer le degré (dénomination du héros avec handicap, lieu de vie, vêture, rôles sociaux) ;

— l'inscription du héros dans son environnement social : relations intrafamiliales, relations avec les pairs ou dans l'environnement scolaire ;

— l'examen de l'issue de l'histoire en tant que le dénouement offre une ouverture possible par un message, une morale de l'histoire qui inviterait le lecteur à se positionner.

\section{RÉSULTATS}

\section{Les supports de l'anthropomorphisation}

\section{Quels types d'animaux?}

Une enquête de Babélio (2012) ${ }^{4}$ portant sur 2400 livres contenant des animaux concluait que dans 2234 d'entre eux (soit 93,1 \% des ouvrages), les mêmes vingt animaux étaient mis

4. http://la culturesepartage.over-blog.com/article-les-20-animaux-de-la-littérature-jeunesse-par-babelio112812188.html 
en scène. Quatre animaux arrivent en tête : le loup (367 ouvrages soit 15\% des 2400 livres), le chien $(12 \%)$, le chat $(11,5 \%)$ et le cheval $(10 \%)$; suivent la souris $(9 \%)$, l'ours $(6 \%)$, le renard $(4 \%)$, le tigre $(3,5 \%)$, le lapin $(2,9 \%)$, puis, à des pourcentages moindres, la grenouille, le crocodile, le singe, l'éléphant, la poule, la vache, la girafe, la chouette et le hérisson. Au final, dans environ la moitié des ouvrages (49\%), les quatre mêmes animaux (loup, chat, chien et cheval) sont représentés, dans $44 \%$ figurent les quatorze animaux « secondaires » (souris, ours, renard, tigre, lapin, grenouille, crocodile, singe, éléphant, poule, vache, girafe, chouette, hérisson) et dans seulement $6,9 \%$ des cas on trouve d'autres animaux.

On ne retrouve pas ces proportions dans notre corpus de trente et un animaux (quatre animaux dans un seul ouvrage [11]); les quatre premiers animaux ne sont présents qu'à cinq reprises : deux loups $[6,25]$, deux chats $[8,10]$, un chien [19] et aucun cheval, ce qui représente $16,1 \%$ de notre corpus versus $49 \%$ de l'étude Babélio.

Seize des animaux dont la fréquence dans l'étude Babélio se situe entre 10 et $2 \%$ sont présents dans onze albums : quatre lapins $[3,7,9,20]$, deux souris $[27,28]$, deux grenouilles $[16,24]$, un ours [21], un tigre [18], et une poule [15], soit 35,5\% de notre corpus versus $44 \%$ de l'étude Babélio.

En revanche, nous avons sept fois plus d'animaux peu fréquents (48,4 \% versus $6,9 \%$ dans l'étude Babélio) : des volatiles (poussin [1], oie [4], mouette [2], hirondelle [17], merlette [22], canard [26]), des animaux marins (zouritte [11], cacatois [11], homard [11], tortue [11], poisson [14]), des mammifères (sanglier [12], hippopotame [13]), ainsi qu'une fourmi [5] et un escargot [23].

Notre corpus comporte donc trois fois moins d'animaux habituellement les plus représentés et sept fois plus d'animaux classiquement moins représentés. Le handicap exigerait-il une différenciation supplémentaire ? Mais, de fait, notre corpus est restreint sur plusieurs dimensions : âge des lecteurs, période de publication, et présence du handicap. 
Selon certains auteurs, « À travers le héros, c'est l'émotion qui parle, l'envie de retrouver un personnage familier, fidèle et aussi rassurant que son nounours. Le héros est un ami du quotidien » (Gombault, Miri, Rabany, 2002, p. 49), ce qui étonne quant à l'identification ou tout du moins l'empathie qu'un jeune enfant pourrait avoir pour un sanglier, une merlette ou une fourmi. Ce bestiaire surprenant nous conduit à nous demander si ces choix ne vont pas à l'encontre de l'objectif visé ; on peut penser que l'option d'un animal peu familier renvoie à une intention délibérée de marquer la différence et de mettre à distance, mais alors ne risquet-on pas de générer l'indifférence de l'enfant qui prend connaissance de ce récit ? Il reste que le capital sympathie n'est pas nécessairement supérieur pour certains animaux habituellement fréquents dans les albums, puisqu'on trouve aussi des souris et des grenouilles.

Une tentative d'établir des liens entre types de handicap et espèces animales ne permet pas d'éclairer ce questionnement; en effet aucune catégorie d'animaux n'est particulièrement porteuse de troubles ou difficultés spécifiques. Pour donner quelques exemples de cette dispersion des types de handicap sur les espèces animales, on peut relever que l'atrophie d'un membre concerne aussi bien une fourmi [5] et une souris [27] qu'un lapin [7] et un chat [10], que les troubles autistiques touchent un poisson [14], un chien [19 ] et un merle [22] et que les troubles de la vue affectent un lapin [3], une grenouille [16] et des souris [28].

\section{Quels types de handicaps?}

L'ensemble des animaux sont nés avec leur handicap, sauf un seul cas d'accident mentionné comme origine du handicap, mais la jeune hirondelle concernée n'était encore qu'oisillon dans son nid [17].

Quatre ouvrages abordent le handicap sensoriel : trois la cécité $[3,16,28]$ et un la surdité [20]. Dans huit livres l'animal ne possède pas les caractéristiques de son espèce, ce qui nuit à 
son développement et ce qui le limite dans sa participation aux activités de l'espèce : un poussin bleu ne parle pas comme les autres [1], un loup gentil n'a qu'une seule dent [6], une zouritte a sept pattes au lieu de huit, un homard a quatre pinces au lieu de deux, une tortue est affublée d'un parasol en lieu de carapace et un cacatois a une bouche à la place du bec [11], un loup est gentil [25] et un canard aboie [26]; huit albums abordent le handicap moteur via des animaux qui ne peuvent pas se déplacer normalement : voler [2, 4, 17, 27], marcher [7, 8, 10] ou accomplir leur rôle avec une patte en moins [5]; onze albums abordent des troubles du développement ou des apprentissages ; quatre une déficience intellectuelle (deux fois une trisomie 21) [9, 18, 21, 23], deux une maladie génétique ayant pour conséquences des difficultés de comportement, cognitives et motrices [12,13], trois l'autisme [14, 19, 22], un trouble du langage [15] et un la dyslexie [24]. Les ouvrages abordant les troubles du développement et des apprentissages sont donc les plus nombreux : s'agit-il des difficultés les plus difficiles à se représenter pour un enfant, le handicap n'étant pas « visible » et engendrant des difficultés de communication et/ou comportementales pour les enfants atteints?

\section{Quel degré d'anthropomorphisme?}

Pour évaluer la nature et le degré d'anthropomorphisation nous avons examiné la proximité ou la ressemblance avec des caractéristiques humaines pour quatre thématiques que nous avons retenues parce qu'elles sont repérables dans tous les albums, dans le texte ou grâce aux illustrations : la dénomination du héros porteur de handicap, la nature de l'habitat, la vêture, les rôles sociaux des protagonistes de l'histoire. D'autres dimensions, comme la richesse des échanges verbaux ou la nature des interactions, n'ont pas pu être prises en compte, car certains albums pour les plus jeunes n'en permettaient pas le codage. 


\section{La dénomination du héros}

Dans quatre albums, les animaux sont simplement désignés comme un petit animal d'une espèce particulière (cacatois, tortue, homard, hirondelle, loup, souris) [11, 17, 25, 28]. Dans tous les autres livres, les animaux héros sont appelés, soit par des caractéristiques qui renvoient à la nature de leur handicap (« Pouet Pouet » désigne l'oiseau qui ne sait dire que cela, « chat loupé » le chat qui a une jambe plus courte que l'autre, «Mimi l'oreille » le lapin à qui il manque une oreille, « Patrovitt» l'escargot plus lent que tous ses congénères), soit par des prénoms (Écume, Borca, Crevette, Neigeuse...), dont certains sont similaires à ceux des humains (Sacha, Manon, William, Chloé, Léonard...) $[1,2,3,4,5,6,7,8,9,10,12,13,14$, $15,16,18,19,20,21,22,23,24,26,27]$.

\section{Le lieu de vie}

Dans douze livres, l'animal vit dans son milieu naturel (dans les bois, dans un terrier, dans la mer...) $[2,3,4,7,11,15,16,17,22,25,27,28]$; dans les seize autres albums l'anthropomorphisme est marqué au minimum par des éléments de décor et mobilier, évocateurs de l'habitat humain (lampe, assiettes, table, lit) ; il est parfois poussé jusqu'à faire évoluer les animaux dans d'authentiques maisons ou appartements situés en milieu urbain ou rural $[1,5,6,8,9,10,12,13,14,18,19,20,21,24,23,26]$.

\section{La vêture}

Dans douze ouvrages l'animal vit dans son état naturel $[2,3,7,10,11,15,16,17,22,23$, $25,28]$. Pour les seize autres albums, l'animal est au moins doté de quelques accessoires (chapeaux, lunettes, tablier, couronne, sac à main...) ou habillé de pied en cap (robes, 
salopettes, vestes, tee-shirts, pantalons...) $[1,4,5,6,8,9,12,13,14,18,19,20,21,24,26$, 27].

\section{Les rôles sociaux}

Dans douze albums, les animaux sont considérés uniquement dans leurs fonctions animales $[2,3,10,11,12,15,16,17,18,25,27,28]$; dans les seize autres, ils ont des statuts professionnels (garagiste, coiffeuse, boulangère, maîtresse, bouchère) dont certains directement articulés aux difficultés dont le héros est porteur (ours médecin qui établit le diagnostic de handicap, pie orthophoniste, écureuil kinésithérapeute, hibou psychologue...) $[1,4,5,6,7,8$, $9,13,14,19,20,21,22,23,24,26]$.

Si les livres offrent une grande variété de conjugaison sur ces seuls quatre thèmes examinés, on peut remarquer que dans quatre albums les protagonistes (des animaux marins, une hirondelle, un loup, des souriceaux) des récits conservent l'essentiel de leur condition animale : ils ne sont désignés que par l'appartenance à leur espèce, vivent dans leur milieu naturel, ne portent aucun vêtement et n'exercent pas de métier [11, 17, 25, 28].

À l'opposé, onze albums $[1,5,6,8,9,14,19,20,21,24,26]$ mettent en scène des animaux qui sont désignés par des petits noms, évoluent dans des habitats dont certains éléments sont clairement empruntés au monde des humains, portent des éléments de vêture ou accessoires caractéristiques des vêtements humains et exercent des professions. Les animaux de ces onze livres sont d'une grande diversité et si on trouve parmi eux un chat, un chien et deux lapins qui peuvent être des animaux domestiques, il y a aussi une fourmi, un poisson, une grenouille, un canard, un oiseau et un ours, animaux moins familiers, dont on ne peut guère s'attendre à ce qu'ils se fondent dans un environnement humain et en adoptent les habitudes. 
Entre ces deux pôles, les treize autres albums présentent des animaux qui associent de diverses façons des éléments propres à leur animalité et des caractéristiques humaines, et on retiendra qu'il n'y a pas de relation entre l'espèce animale et le degré d'anthropomorphisme évalué à travers les quatre thématiques retenues.

Au final, les tendances essentielles peuvent être résumées ainsi : le jeune héros est le plus fréquemment doté d'un surnom ou prénom $(n=24)$; il évolue dans un environnement soit considéré comme spécifiquement animal, soit très proche de l'environnement humain $(\mathrm{n}=12$ vs 16); il est représenté à l'état «naturel» ou doté de quelques accessoires et vêtements qui l'humanisent ( $\mathrm{n}=12$ vs 16), humanisation parfois renforcée par l'attribution de rôles sociaux ( $\mathrm{n}=16$ vs 12). De façon générale, on peut donc considérer que la composante identificatoire animale reste marquée et qu'elle s'adjoint, à des degrés divers, d'une ou de quelques composantes qui viennent signifier son humanité, essentiellement le prénom comme appellation ou comme terme adresse - composante individuelle -, secondairement par le lieu de vie, la vêture ou les rôles sociaux, tendance qui semble se conforter dans les ouvrages les plus récents. En effet, les livres dans lesquels le degré d'anthropomorphisation est le plus élevé sont récents (un de 2012, un de 2015, et trois de 2016) ${ }^{5}$.

\section{Les relations entre le héros avec handicap et son réseau familial et social}

\section{Les réactions négatives des autres personnages}

Les réactions les plus fréquemment rencontrées sont les moqueries des pairs d’âge mentionnées dans la moitié des ouvrages : moqueries envers le petit animal en situation de

\footnotetext{
5. Notre corpus d'analyse s'arrête à 2016. Deux albums du corpus ont été réédités en 2017 [5 et 9] et ces
} versions nouvelles s'avèrent beaucoup plus anthropomorphisées que les versions précédentes. 
handicap $[1,3,4,6,7,8,9,10,11,13,16,23,24,25]$, que ce soit de la part de la fratrie $[3,7$, 25] : «Ce pauvre lapin louchait et les deux autres n'arrêtaient pas de se moquer de lui » [3], « Jojo éclate de rire parce que Lapinou n'avance pas d'un pouce » [7], des camarades de classe $[1,8,23,24]$ : «Toute la classe éclata de rire » [1], « Tous les élèves de la classe se moquent de Patouille ! - Hé, Patouille, il ne sait pas lire ! - Il devrait retourner en maternelle ! » [24] ou des pairs, congénères ou autres petits animaux $[4,6,9,10,13,16]$ : « Hélas, ils subissaient souvent les moqueries du chat Huteur et sa bande d'affreux mistigris », «Tu louches ! Tu louches ! crie le martin-pêcheur en la voyant » [16], qui peuvent chanter des chansons moqueuses $[1,3,9]$ : « Ils lui chantaient sans cesse : "Regarde à droite pour voir à gauche. Regarde en haut pour voir en bas. Ah-ah-ah, ah-ah-ah” » [3], « Hou hou, Doudou-Lapin-petit-boudin-qui-comprend-rien ! » [9], utiliser des sobriquets [8] comme Chatmallow, imiter le jeune héros dans ses difficultés liées au handicap [10] : «Ils les imitaient en riant comme des ouistitis, se tapaient dans la patte, fiers de leurs âneries » et utiliser quolibets et insultes $[11,23]$ : « chose ridicule » [11], « Oh là là, quel traînard ! » [23].

Les réactions de rejet sont aussi fréquentes puisque présentes dans neuf albums $[4,5,6,13$, $14,16,17,21,26]$, mais elles concernent tant les pairs que les adultes : rejet à l'école de la part des camarades de classe $[14,26]$ : « Pousse-toi Hercule, tu prends toute la place avec ta bulle !» [14], « Ses camarades refusent de nager avec lui, sous prétexte qu'il est trop... “bizarre” » [26], mais rejet qui peut venir aussi de la sœur du héros [21], des camarades [16], des humains [6], de la reine [5] qui n'éprouve pour la fourmi infirme que pitié dégoût et condescendance : «Otez-la de mon regard. Ça me rend triste et quand je suis triste je n’arrive plus à pondre ». Mère et congénères peuvent être indifférents [4] : «Personne ne remarqua son absence »; mère, sœurs et toutes les hirondelles délaissent la petite hirondelle et migrent sans elle du fait de son infirmité [17] ; maîtresse ou directeurs d'école [13, 21] peuvent enfin être eux aussi à l'origine du rejet : « Dans mon école, les élèves doivent être musclés et 
sportifs. Je suis sincèrement désolé d'avoir à te dire ça, mais tu ne corresponds pas vraiment à ces critères... » $[13]$.

Dans les trois livres sur l'autisme $[14,19,22]$ des sentiments particuliers sont mis en avant : des pairs qui paraissent interloqués : «Hercule, sors un peu de ta bulle ! » [14], « ils sentaient bien que Ted était différent et ils se posaient beaucoup de questions » [19], se sentant eux-mêmes rejetés : « Silus était bien triste ; il pensait que Ted ne l'aimait pas » [19], et un frère qui a peur : «Merli, alors poussait des pépiements d'épouvante. Ses parents devaient l'emmener dans le nid voisin » [22].

Mentionnons enfin l'énervement, le refus de la fratrie de surveiller les petits sangliers avec handicap [12], le jugement de réprobation des poules adultes qui considèrent cette poulette mal élevée [15], la maîtresse qui gronde le petit escargot trop lent ou le père qui est à la fois déçu, énervé et fâché [25] : «Tu es copain avec les lapins, grondait Papa de sa grosse voix et tu n’as jamais attrapé le moindre petit poisson ! Petit Loup, tu nous désespères, qu'allonsnous faire de toi ?». La honte des parents $[11,15]$ est aussi présente.

\section{Les réactions familiales empathiques}

Les parents se montrent en premier lieu souvent choqués, inquiets et tristes $[7,11,15,17$, $18,19,21,22,23,25]$, abasourdis par le diagnostic : «Tout s'écroule dans la tête de Mamili et Papalapi » [7], « Papa ours est resté là, son bouquet de fleurs à la main » [21], « Abasourdis, monsieur et madame Merlo n'étaient plus capables de la moindre pensée » [22] ; inquiets tant de façon immédiate : « Maman poule et papa Kocoriqui sont très inquiets » [15], que pour l'avenir [23, 25]. Ils sont également compréhensifs, bienveillants, patients et investis dans l'éducation de leurs enfants avec handicap [12, 18, 21, 22, 25, 26]. 
La fratrie de son côté apparaît à la fois également inquiète $[7,12]$ et coupable d'avoir ri [7] ou de s’être dérobée à la surveillance de leurs puînés avec handicap : « Trop fier pour l'avouer, le jeune sanglier était lui aussi mort d'inquiétude pour son frère et sa sœur » [12].

Dans vingt et un ouvrages, soit deux tiers du corpus, l'entourage est soutenant $[1,2,4,6,7$, $8,9,10,11,13,14,15,16,19,20,21,22,23,26,27,28]$.

Dans neuf albums $[2,4,8:$ handicap moteur $][9,19,21,22,23,24:$ troubles du développement], l'entourage encourage le héros avec handicap dans ses apprentissages pour l'aider à progresser : le bernard-l'hermite encourage la petite mouette et lui fabrique une prothèse pour qu'elle puisse voler [2], un ami oie apprend patiemment à Borka à nager et l'accepte telle qu'elle est [4], un grand pigeon aide la merlette à voler [22].

On trouve également un encouragement indéfectible de la part des parents $[8,9,21,22,24]$ qui consultent différents spécialistes, soutiennent leur petit, ne baissent pas les bras malgré les difficultés et l'aident à avoir confiance en lui : «Papa Ours aussitôt s’accroupit et fait de ses bras un rempart de château fort autour de son ourson » [21], « Maman et moi, nous sommes à tes côtés. Nous t'aimons et nous ferons tout pour t'aider », « Aie confiance en toi, et tu verras, ça va aller de mieux en mieux » [24]. Les enseignants contribuent à soutenir les efforts des petits animaux en difficulté : " grâce à la patience et à la bonne volonté de tous, Ted apprenait à vivre dans la classe comme un autre petit chien. Il faut dire que monsieur Bouvier Bernois et mademoiselle Saint-Bernard savaient comment s’y prendre avec lui » [19], « Tu vois Patouille, tu as trouvé ton talent. Tu dessines magnifiquement bien. Tu peux être fier de toi ! » [24].

Dans neuf livres, l'entourage est bienveillant et ouvert à la différence $[6,7,13,14,15,16$, 20,26], qu'il s'agisse d'un humain qui recueille le petit loup sans dents et s'adapte en lui donnant du steak haché [6], des parents et fratrie qui encouragent le petit canard à aller voir le monde tout en lui disant qu'il va leur manquer [26], d'un directeur d'école empathique et 
ouvert [13], ou d'une étoile de mer acceptant le petit poisson comme il est en allant vers lui : « Bonjour, je m’appelle Esther», déclare l'étoile de mer à Hercule. « Waouh, elle est vraiment chouette ta bulle ! » [14].

Dans deux ouvrages, suite aux moqueries, un petit animal prend la défense du héros avec handicap ; son camarade de classe prend la défense de Pouet-Pouet : « Ce n’est pas drôle du tout ! protesta Mimie » [1]; Lili prend la défense de son petit frère Doudou lapin : « Alors Lili leur crie qu'elle les déteste et que c'est eux, les gros boudins qui ne comprennent rien !» [9].

On peut enfin relever une entraide que les petits animaux avec handicap vont s'apporter les uns aux autres $[10,11,26,27,28]$, mêlant leurs différences pour se sentir plus forts encore : « Depuis ce jour, la petite tortue et la petite zourite, le cacatois vert et le homard à quatre pinces vivent ensemble en communauté et on n'a jamais vu meilleure entraide !» [11].

\section{Réaction aux réactions : les ressentis du héros avec handicap}

Dans dix albums $[7,9,12,15,18,19,21,22,27,28]$ les sentiments des héros vis-à-vis de leur handicap ou des réactions des autres ne sont pas abordés, majoritairement pour les héros avec un trouble du développement ou des apprentissages [9, 12, 18, 19, 21, 22]. Soit aucun sentiment du héros n'est mentionné $[15,18,21,28]$, soit le jeune héros ne semble pas se rendre compte des réactions de ses pairs $[9,19,22]$ : « Ses yeux restaient dans le vague et on voyait parfois son regard se défaire » [22], même si pointent des moqueries [9], ou du danger [12]; il peut même se montrer content $[7,27]$ : « content de découvrir le monde avec ses frères et sœurs, il rit » [7].

Les sentiments que l'on retrouve le plus souvent sont la tristesse et la solitude. 
Dans treize livres, la solitude et l'isolement du héros sont mentionnés [1, 3, 4, 8, 10, 11, $13,14,16,17,24,25,26]$. Certains vont jusqu'à se réfugier dans le terrier [3], partir ou s'enfuir $[10,11,25,26]$ : « Tout cela rendait Petit Loup si triste qu'il s'enfuit dans la forêt profonde » [25]. Ils peuvent être repliés sur eux-mêmes [1, 3, 14, 17] : « Alors, il reste seul, enfermé dans sa bulle, à l'abri du monde extérieur » [14], sans amis [1, 8, 10, 16, 24], les autres animaux ne voulant pas jouer avec eux : «Pouet-Pouet ne jouait plus avec personne» [1], « Il rêverait tant d'avoir une super bande de copains... » [8], « les autres élèves ne le remarquent même pas » [24], « Puisque personne ne veut jouer avec lui » [26], entraînant un sentiment de rejet : « Aucune école ne veut de moi. Je suis trop petit, trop gros et trop bête pour y entrer » [13], ou même d'abandon de tous [4].

Dans douze albums (presque la moitié) $[1,2,4,5,8,10,11,13,16,17,24,25]$ le héros est triste, voire malheureux : «À l'école les jours étaient devenus tout gris » [1], « Et le petit homard était bien malheureux ! » [11], « Patouille est très triste » [24], « La petite hirondelle se recroquevilla à l'intérieur et se mit à trembler de peur et de chagrin » [17] et des pleurs sont mentionnés $[1,2,4,5,13,16,25]$ : «Une grosse larme coula sur sa joue » [1], « Ecume n'en a pas fini de pleurer » [2], « Les larmes dégoulinaient le long du bec de Borka » [4], « Il va s'asseoir sur un rocher, non loin de là, et se met à pleurer à chaudes larmes » [13].

Dans cinq ouvrages, le héros avec handicap se sent complexé $[5,13,20,24,25]$ : « Je ne suis pas comme les autres, je suis laide avec cette patte en moins » [5], « Mais ce qui complexe et énerve le plus William, c'est qu'il a du mal à apprendre et comprendre ce qu'on tente de lui enseigner » [13], « Je pleure parce que je ne suis pas comme il faut !» [25].

Dans quatre ouvrages, le héros avec handicap montre une force de caractère pour dépasser son handicap $[5,8,20,23]$, notamment en faisant plus d'efforts que les autres : «Pendant que les autres se reposent, il prend des cours du soir pour rattraper son retard » [23] ou en se défendant : « Non! Ça ne se passera pas comme ça ! », «Grosse Madame la Reine, ça m’est 
égal que vous n'aimiez pas me voir! Moi, je n'aime pas tellement vous voir non plus » [5]. À deux reprises, le héros avec handicap est irrité de ne pas arriver à faire comme les autres [1, 6] : «Vexée et triste à la fois, la petite mouette finit par s'isoler derrière un rocher » [1] et peut se fâcher : « La colère lui monte au nez et il réduit en confettis un sac-poubelle égaré ». [6].

\section{Le dénouement de l'histoire et son « message »}

Le dénouement de l'histoire permet de faire passer un message, une morale qui invite le lecteur à se positionner.

\section{Du handicap compensé au super héros}

Dans quatre ouvrages, le héros avec handicap devient super héros $[1,2,3,8,26]$. Pour deux, cela fait suite à une compensation ; ainsi la petite mouette [2] avec ses prothèses aux ailes devient la plus rapide et la différence au sein de la fratrie est ainsi gommée : « Désormais, quand les trois sœurs organisent un concours de vitesse, Ecume se montre la plus rapide »; le petit chat [8], inventeur de sa propre prothèse, saute maintenant mieux que les autres au trampoline et fait l'admiration de tous : « Tous les chats miaulèrent d'admiration : Sacha, Sacha, montre-nous comment tu fais ! Apprends-nous à rebondir comme toi !». Quant au petit canard [26], le fait qu'il ne présente pas les caractéristiques de son espèce puisqu'il aboie, provoque la fuite du voleur et l'admiration générale : «Ses parents, émus, le serrent dans leurs ailes tandis que ses camarades, admiratifs, l'accueillent à présent comme un héros »; le petit poussin [1], pousse un fameux Pouet-Pouet (une des caractéristiques de son handicap) ce qui fait fuir le méchant poussinator, il sauve ses camarades d'une mort certaine et devient héros plébiscité : « Le soir même, l’exploit de 
Pouet-Pouet fit le tour de toute la ferme ; même Flabard Dubec applaudissait ! ». L'animal avec handicap peut aussi devenir héros malgré lui [3], ainsi le lapin loucheur mord la queue du renard en pensant que c'est une carotte, sauve ses frères et suscite leur admiration : « sans toi cette sale bête nous aurait mangés » et leur acceptation « Maintenant, ils s'entendent très bien tous les trois ».

\section{Le changement de regard sur le héros avec handicap de la part de l'entourage}

Dans neuf albums, le changement de regard sur le héros lui permettra de mieux être accepté ou de mieux s'accepter lui-même, sans qu'il y ait d'évolution pas rapport à la situation de handicap. Le changement de regard peut concerner :

— les pairs [10], comme c'est le cas du chat Huteur qui se moquait du handicap du chat Loupé, jusqu'à une chute après laquelle seul le chat Loupé lui tend la patte : «Cette aventure fit comprendre au chat Huteur qu'il n'y a rien de bien à jouer les moqueurs » ;

— un directeur d'école [13] qui, contrairement à d'autres directeurs, est bienveillant et accueille tous les petits animaux dans leur diversité : «Dans mon école, tous les animaux sont acceptés avec leurs différences » ce qui permettra à William l'hippopotame de se faire des amis ;

— les parents, parents sangliers [12] dont le regard évolue quant aux conséquences du handicap de leurs petits et de la charge reposant sur les épaules de la fratrie et les parents de Petit Loup [25], qui après avoir été tristes et en colère évoluent dans leur appréciation :

« Regarde nos enfants, dit Papa Loup à Maman Loup, ils se ressemblent tous, et pourtant tous sont différents. [...] Et Petit Loup, s'écria Maman Louve, il est unique ». Le changement de regard peut être aussi du petit animal avec handicap sur lui-même, qui lui permettra de mieux s'accepter, ce qui est le cas de Chloé, la petite grenouille [16] qui se sent en situation de 
réussite, d'Ignace [6] qui après avoir voulu ressembler à un chien n'ayant pas les caractéristiques d'un loup, finit par s'accepter tel qu'il est : « Ignace ne gagnera jamais le concours des Canines, mais il est fier d'être un "loup à une dent" », de Mimi l'oreille [20] qui après de vaines tentatives pour faire remplacer son oreille : « décida donc de rester comme il était » et de Bélem [21] à qui la tache en moins ne manque plus puisqu'il sait maintenant la trouver partout : « dans tout ce qu'il aime, dans les yeux de son papa, de sa maman, de sa grande sœur, dans le regard d'un chat ». Dans un ouvrage [24], le changement de regard sur le handicap s'effectue à la fois par les pairs de la petite grenouille, suite à un dessin apporté en classe à la demande de la maîtresse, qui veut que chacun parle de ses talents : « Pourtant, cette fois, personne ne se moque de lui. Le dessin est si joli que Patouille n'a même pas besoin de parler. Tous les élèves de la classe se lèvent et applaudissent » et aussi sur elle-même puisque cela lui donnera confiance en ses capacités.

\section{Changement de regard sur le héros induisant une évolution des compétences}

Dans sept ouvrages, le changement de regard des autres permettra au héros de progresser dans ses compétences, la situation de handicap, n’est jamais annihilée, mais réduite ou compensée. Cela peut être un changement de regard des pairs à l'école, grâce notamment à une demoiselle Saint-Bernard [19], AESH dont il a été décidé qu'elle « veillerait sur Ted pour qu'il puisse être intégré avec les autres chiens de son âge et accepté avec sa différence », ce qui aidera le chiot à progresser ou grâce à une camarade de classe du petit poisson [14] qui cherchera à faire sa connaissance malgré sa différence en rentrant dans sa bulle : « Pour la première fois de sa vie, quelqu'un apprécie la bulle d'Hercule », ce qui permettra à Hercule d'entrer en contact avec autrui. Une petite oie [4] qui était rejetée par de premiers camarades en rencontre d'autres qui ont un regard différent : « Il importait peu aux oies de Kew Gardens que Borka n'ait pas de plumes », non seulement la jeune oie pourra avoir de nouveaux amis et 
être heureuse, mais aussi apprendre à nager. Changement de regard également des parents, dont ceux d'un petit tigre [18] qui en donnant plus de temps à leur petit et en étant patients l'aideront à progresser : « Il peut dire toute une phrase, et cette phrase c'est... Moi aussi, je sais le faire », ou ceux d'une petite poule [15] qui, en s'armant de patience sous les conseils de la reine des pondeuses, soutiendront ses progrès : « Papa Kocoriqui et maman poule ne sont plus inquiets : Crevette sait parler. Il ne lui reste plus qu'à apprendre à bien parler ». Le regard des parents d'une merlette [22] évolue et leur permet de continuer à se battre pour l'oisillon : « Soudain, son père la voyait autrement, tel son oisillon, malade peut-être, mais vivante. Elle était sa fille, Neigeuse... Alors, il résolut de rebâtir le nid » qui progressera : « Neigeuse vola seule, un peu plus tard. Tant d'obscures peurs l'habitaient, qui, sans arrêt ressurgissaient, qu'elles furent longues à se défaire. Mais enfin, elle vola ». À son tour le regard des parents de Doudou-lapin [9] évoluera, grâce à sa petite sœur ; les parents s'engageront alors dans un processus de stimulation et un accompagnement par divers professionnels qui aideront leur enfant à progresser « Doudou-Lapin parle presque très bien. Il va même peut-être aller bientôt à la petite école des lapins ».

\section{Le héros avec handicap prend une place spécifique}

Dans six ouvrages $[5,7,11,23,27,28]$ le handicap est toujours présent, mais il y a un changement de place, le héros prend alors des fonctions spécifiques. Dans quatre ouvrages, les héros mêlent leurs différences pour s'entraider et se sentir plus forts. Pour trois d'entre eux, il y a entraide entre différents animaux héros : le fait de partager leurs informations permettra aux petites souris [28] de deviner, malgré leur cécité, que ce sur quoi elles grimpent est un éléphant, le souriceau [27] s'allie, pour s'en sortir, à d'autres animaux ayant d'autres difficultés : «Un seul d'entre nous n’y serait jamais arrivé sans les autres », et la Zouritte, le Cacatois, le Homard et la Tortue [11] fonctionnent ensemble pour suppléer le handicap de 
chacun d'eux : «Le parasol de la tortue leur sert de maison à tous et c'est la Zourite qui le tient elle qui a tant de pattes ! Le petit homard gris prépare à manger pour tout le monde, il s'aide de toutes ses pinces et le travail est vite fait ! Le cacatois n'a plus de problème pour manger! ». Lapinou [7] et ses frères et sœurs conjuguent leurs compétences pour trouver des aliments : Lapinou ne peut pas se déplacer, mais il a un très bon flair : « Lapinou, son flair étonnant, Jojo, l'ingéniosité..., ils mêlent leurs différences pour se sentir plus forts encore ». Dans les deux autres ouvrages, les héros vont trouver un emploi adapté à leurs difficultés et une place spécifique, Mina la petite fourmi [5], avec sa force de caractère et son courage, travaille à la nurserie pour s'occuper des bébés fourmis différents : « Si jamais vient au monde une petite fourmi à qui il manque une patte, comme à moi, ou une antenne, ou une mandibule... que saurez-vous lui dire? Moi, je lui expliquerai qu'en dedans, elle est tout à fait comme les autres. Et moi, elle me croira »; Patrovitt l'escargot devient chauffeur-livreur de graines qui, grâce à sa lenteur, sont devenues fleurs quand il arrive à destination, ce qui fait gagner du temps aux jardiniers.

Le handicap est toujours présent et n'évolue pas au cours de l'histoire, ce sont les petits animaux qui vont se servir de leurs différences pour trouver leur place, y occuper des fonctions spécifiques et être utiles aux autres.

\section{DISCUSSION}

\section{L'anthropomorphisation et ses supports}

En examinant les types d'animaux et de handicaps et leur degré d'anthropomorphisation, nous avons d'abord analysé les supports formels de l'identification supposée ou alléguée.

La diversité animale est marquée, et deux hypothèses ont été formulées pour en rendre compte, sans pouvoir trancher : s'expliquerait-elle davantage par la jeunesse du public, les 
auteurs l'imaginant plus accessible à cette diversité que des enfants plus âgés plus imprégnés déjà de catégorisations culturelles, ou bien la thématique du handicap serait-elle d'autant plus acceptée que le support serait plus éloigné de la réalité humaine et il s'agirait alors de stigmatiser le héros porteur de handicap pour souligner la différence?

Nous avons observé une plus forte représentation des troubles du développement et des apprentissages : sans doute s'agit-il là d'une évolution éditoriale récente qui marque l'intérêt du recours à la littérature jeunesse en tant qu'étayage aux situations d'apprentissage et de socialisation.

L'enfant est invité au jeu de l'identification d'abord par les jeux d'appellation et d'adresse, plus discrètement ensuite par la présence d'accessoires ou d'éléments de l'environnement marquant son caractère humain. L'attribution de rôles sociaux, degré le plus « anthropomorphisant » d'une identification supposée, est présent dans plus de la moitié des ouvrages et les animaux ont volontiers des statuts professionnels, la plupart du temps liés au handicap (professionnels du diagnostic, des soins médicaux, paramédicaux ou de l'enseignement ; l'anthropomorphisme social le plus poussé - trois ouvrages - a d'ailleurs trait à ces derniers).

Les auteurs plébiscitent les animaux et, en introduction, nous avons évoqué plusieurs sources d'explications pour rendre compte de cette orientation. Reprenons deux axes essentiels : le passage par l'animal permet d'octroyer au récit un supplément d'universalité ; en dépassant un cadre social ou géographique, on accéderait à une forme d'intemporalité ; en éloignant le récit de la réalité tangible dans laquelle baigne le lecteur, l'abord de thèmes plus difficiles (la violence, la mort, ici le handicap) serait facilité du fait d'une moindre violence. Le «message » déployé, dès lors, serait d'autant plus efficace qu'il s'appuie sur des histoires d'animaux (Leprince, 2017). Quant à l'universalité, Gaiotti (2013) fait remarquer qu'en 
décrochant l'histoire de son ancrage animal d'origine pour l'insérer dans un cadre plus « familial », on a souvent maintenu des représentations traditionnelles, en particulier sur le genre (la présence massive des animaux distingués en fonction du critère mâle ou femelle et des attributs supposés des uns ou des autres a eu pour effet de maintenir les stéréotypes). Toutefois, en gommant les composantes « sociales », comme le font nombre d'ouvrages destinés aux plus jeunes, la mise à distance impliquée pourrait rendre d'autant plus complexe le jeu d'identification que l'animal ne dispose pas d'un capital sympathie évident... pas si sûr que l'enfant saisisse la leçon (voir ci-dessous Larsen, Lee, Ganea, 2017).

Mais, bien évidemment, il ne s'agit ici que d'éléments de contexte facilitant ou non le message supposé, tel qu'il s'exprime au travers des relations que le jeune héros construit avec son environnement familial, amical, social.

\section{Les relations avec l'entourage}

Nous avons observé que dans la moitié des ouvrages du corpus, les réactions négatives des «autres » sont présentées de façon clivée (moqueries, rejets...), et invitent le lecteur à se positionner contre ses moqueurs. Les enfants-héros sont, d'un autre côté, soutenus et encouragés par leur famille, parfois aussi par l'entourage social ouvert à la différence, voire aidés par les pairs qui prennent leur défense. Mais, dans plus d'un tiers du corpus, le ressenti du jeune porteur de handicap n'est pas abordé. Lorsqu'il est évoqué, solitude, tristesse, à un moindre degré ressenti de «complexe», apparaissent comme les émotions les plus fréquentes, alors que les expressions de force de caractère pour dépasser le handicap sont nettement plus rares.

Weisser (2004) évoque deux types d'ouvrages, ceux qui entraînent un positionnement clair, clivé, des enfants lecteurs qui vont se rallier au «gentil» héros et à sa cause, contre les 
« méchants » enfants moqueurs et rejetant, et ceux dont le texte est ouvert n'indiquant pas la position de l'auteur sur les ressentis et émotions des personnages par rapport à leurs comportements et conséquences, ce qui permettrait une « compréhension de la complexité et richesse des comportements humains et de leur ambivalence » (p. 129). Pour lui, les premiers peuvent certes faire adhérer les lecteurs à la valeur de tolérance, mais il se questionne sur le fait que cela ait un effet sur leurs comportements. Alors que les seconds permettraient une réflexion plus approfondie, moins clivée et critique, plus ambivalente et tolérante, donc plus de résonnance subjective : être plus bienveillant que critique et, peut-être, influencer les comportements. On peut donc se questionner quant à l'impact de ces ouvrages clivés à propos du handicap sur l'évolution des comportements des enfants lecteurs.

\section{Le dénouement de l'histoire comme « morale»}

Nous avons mis au jour quatre modalités qui permettent au jeune héros d'évoluer sous le regard de son entourage dans un processus dynamique :

— le handicap apparaît « compensé » quand le héros devient un super-héros, ce qui entraîne un changement du regard des autres;

— la démarche réflexive de l'entourage sur le handicap suscite le changement ;

— le changement de regard permet au héros de progresser, ce qui suscite en retour, par cercle vertueux, une évolution des relations ;

— le handicap est toujours présent, mais les jeunes animaux se servent de leur différence pour trouver leur place, occuper des fonctions spécifiques et être utiles aux autres.

L’objectif général est sans doute de sensibiliser les enfants au handicap, de mettre en mots leurs capacités d'observation, leurs ressentis et leurs appréhensions, pour, ensuite, les rendre 
plus tolérants, bienveillants, initiant des conduites prosociales lorsqu'ils rencontrent un enfant avec handicap.

On peut alors revenir à l'une de nos interrogations initiales relative à l'usage de ces livres en milieu scolaire, d'autant que dix des albums du corpus, soit plus d'un tiers, mentionnent ou abordent la scolarisation, voire en font un sujet central $[1,8,9,13,14,19,21,23,24,26]$. Dans ces ouvrages, l'évolution favorable du héros est très largement conditionnée par le changement de regard de ses pairs, encouragé par des professionnels attentifs à permettre à l'animal en difficulté de développer ses talents et de prendre confiance en soi. On pourrait recommander que de tels livres soient proposés aux enfants des classes maternelles, en particulier lorsque des enfants différents y sont accueillis, ce qui est aujourd'hui de plus en plus souvent le cas. La discussion qui devra poursuivre la lecture de l'album pourrait permettre de saisir comment les enfants s'emparent de la thématique et à quelles transpositions éventuelles elle les conduit.

Nous pouvons nous questionner sur le type de dénouement eù losque le handicap du héros peut s'annihiler et le transformer en super héros, dénouement fort peu probable dans la réalité, donc avec une identification difficile pour de jeunes enfants au contact d'un pair avec handicap. Mais la tolérance et l'empathie sont abordées dans les trois premières catégories, puisqu'elles ont en commun le changement de regard sur la situation de handicap. En effet, dans la première catégorie, le héros devient super héros (soit le handicap est compensé, soit il devient une sorte de super pouvoir), ce qui change le regard des autres sur le petit animal avec handicap vu comme un héros. Dans la deuxième, le regard des autres change, ce qui permet une meilleure acceptation du héros avec handicap par les autres ou lui-même. Dans la troisième, le regard des autres change et permet de faire évoluer la situation de handicap. On peut penser que cela permet de sensibiliser l'enfant au fait que la tolérance, à travers ses comportements et son regard sur ses pairs avec handicap, peut avoir un impact important, et 
que cette acceptation suscite, en retour, confiance en lui-même et progrès pour le héros porteur de handicap. Dans la quatrième catégorie, la notion d'entraide est abordée, le fait que chacun peut occuper une place et des fonctions spécifiques et être utile aux autres. Un dénouement de l'histoire réaliste et proche des valeurs d'inclusion (tolérance, empathie, se mettre à la place de l'autre, entraide) invite à une sensibilisation plus marquée.

\section{CONCLUSION}

Si l'on revient à notre question de départ qui porte sur l'anthropomorphisation et ses effets attendus, ceux-ci sont supposés être doubles : les enfants seraient naturellement attirés vers eux et, par conséquent, en usant d'animaux au comportement anthropomorphique, l'histoire serait plus captivante et ses leçons davantage accessibles aux jeunes lecteurs. Nous avons, d'une part, tenté de montrer que les représentations et les degrés de l'anthropomorphisation étaient divers et complexes, et on ne peut présupposer des effets simples et linéaires, même si l'on s'en tient à ce que nous avons appelé l'anthropomorphisation formelle. Mais sur ces bases l'enfant doit être sensible aux émotions éprouvées et adhérer (ou non) aux morales proposées. La référence à l'animal favoriserait-elle les comportements prosociaux, ceux qui impliquent que l'on se préoccupe d'autrui ? De fait, fort peu de recherches ont exploré cette question. Ganea, Canfield, Simons-Ghafari, Chou (2014) fournissent un indicateur indirect en montrant que les enfants apprennent moins de faits sur les vrais animaux à partir d'histoires qui anthropomorphisent les animaux que d'histoires qui dépeignent les animaux de façon réaliste. Larsen, Lee et Ganea (2017) ont assuré la lecture de trois types d'histoires auprès d'enfants de quatre à six ans. La première est celle d'un petit raton laveur, avec des personnages très anthropomorphiques qui se comportent de façon généreuse avec les autres. La deuxième reprend littéralement la première, mais avec des visuels d'êtres humains. La troisième situation contrôle parle de planter et de contrôler l'évolution de graines, sans 
allusion à des comportements généreux. On indique aux enfants qu'un enfant de leur âge, resté anonyme, a été choisi pour ne pas participer à l'expérience, mais les sujets lecteurs pouvaient partager avec eux des autocollants. L'histoire choisie traite du problème de l'échange et invite à penser que partager avec les autres permet de sentir mieux. Or, c'est la version avec les êtres humains qui suscite le taux le plus élevé de partage d'autocollants. Le transfert d'informations et de comportement serait plus efficace pour de jeunes esprits avec des modèles humains ? Ces seuls travaux, en particulier ici le second, très proche des questions posées, n'explorent qu'une situation spécifique. Ces questions interrogent, d'autre part, non seulement les effets supposés de la lecture, mais ceux-ci sont à examiner dans les contextes de lecture et les effets induits par les pédagogues, dans une société en évolution visà-vis de l'inclusion des enfants en situation de handicap.

\section{RÉFÉRENCES}

Armengaud (Françoise).- Enfants et animaux dans la littérature jeunesse, dans Marcelli (D.), Lanchon (A.), L'enfant, l'animal, une relation pleine de ressources, Toulouse, Erès, 2017, p. 187-208.

Daeninckx (Didier).-Le chat de Tigali, Paris, Mini-Syros, 2007.

Diament (Nic).- Histoire des livres pour les enfants du Petit Chaperon rouge à Harry Potter, Paris, Bayard, 2008.

Gaiotti (Florence).- Présences animales dans les mondes de l'enfance, Cahiers robinson, $34,2013$.

Ganea (Patricia), Canfield (Caitlin), Simons-Ghafari (Kadria), Chou (Tommy).- Do cavies talk? The effect of anthropomorphic picture books on children's knowledge about animals, Frontiers in Developmental Psychology, 5, 283, 2014. 
Gombault (Ludovic-Jérôme), Miri (Nadia), Rabany (Anne).- Littérature : l'album, Paris, Bordas, 2002.

Gravillon (Isabelle).- Ma puce, mon p'tit loup... Des surnoms animaliers pour l'enfant, dans Marcelli (D.), Lanchon (A.), L'enfant, l'animal, une relation pleine de ressources, Toulouse, Érès, 2017, p. 179-185.

Joselin (Laurence).- Filles/garçons : quelles images des héros en situation de handicap dans la littérature de jeunesse? dans Ciccone (A.), Korff-Sausse (S.), Missonnier (S.), Salbreux (R.), Scelles (R.), Handicap, identité sexuée et vie sexuelle, Ramonville St-Agne, Érès, 2010, p. 171-185

Joselin (Laurence).- Représentations plurielles des héros en situation de handicap dans la littérature de jeunesse, dans Schneider (B.) et Mietkiewicz (M.-C.), Des enfants dans les livres : représentations, savoirs, normes, Toulouse, Érès, 2013, p. 103-115.

Larsen (Nicole), Lee (Kang), Ganea (Patricia).- Do storybooks with anthropomorphized animal characters promote pro-social behaviors in young children? Developpemental Science, 2017, p. 1-9.

Lemoine (Lise), Mietkiewicz (Marie-Claude), Schneider (Benoît).- L'enfant porteur de trisomie 21 et sa fratrie dans la littérature jeunesse, Revue francophone de la déficience intellectuelle, 23, 2012, p. 43-55.

Lemoine (Lise), Mietkiewicz (Marie-Claude), Schneider (Benoît).- Fratrie et handicap (trisomie 21 versus autisme) : les représentations dans la littérature jeunesse, Revue francophone de la déficience intellectuelle, 27, 2016a, p. 141-162.

Lemoine (Lise), Mietkiewicz (Marie-Claude), Schneider (Benoît).- L'autisme raconté aux enfants : la littérature jeunesse, un support de sensibilisation pertinent? Enfance, 2, 2016b, p. 231-245. 
Lemoine (Lise), Mietkiewicz (Marie-Claude), Schneider (Benoît).- Enfants en situation de handicap et scolarisation en milieu ordinaire : quelle place pour la famille ? Une approche par la littérature jeunesse, Psychologie \& Éducation, 4, 2017, p. 29-52.

Leprince (Chloé).- Quand les enfants découvriront-ils qu'un écureuil ne va pas à l'école? https://www.franceculture.fr/litterature/anthropomorphisme_le 3-12-2017.

Léon (Renée).- La littérature de jeunesse à l'école, Paris, Hachette, 2007.

Marriott (Stuart).- Red in tooth and claw? Images of nature in modern picture books.

Children's Literature in Education, 33, 2002, p. 175-183.

Nièvre-Chevrel (Isabelle).- Introduction à la littérature de jeunesse, Paris, Didier, 2009.

Perrin (Agnès).- Quelle place pour la littérature de jeunesse à l'école ? Paris, Retz, 2001.

Poslaniec (Christian).- Des livres d'enfant à la littérature de jeunesse, Paris, Gallimard, 2008.

Savier (Lucette).- À chaque layette un livre! dans Causse (R.), L'enfant lecteur. Tout pour faire aimer lire, Paris, Autrement, 1988, p. 65-75.

Weisser (Marc).- Apprendre la tolérance grâce au texte littéraire : de la compréhension littérale à l'interprétation axiologique, Mediterranean Journal of Educational Studies, 9, 1, 2004, p. 121-134.

\section{ANNEXE - CORPUS}

[1] Bertrand (Pierre).- Pouet Pouet, Paris, L'École des loisirs, 2009, 2010.

[2] Boncens (Christophe).- Au bord de la mer... La mouette aux trop petites ailes. Kerangwenn, Beluga Coop Breizh, 2010. 
[3] Boujon (Claude).- Le lapin loucheur, Paris, L’École des Loisirs, 1984, 1986, 2002.

[4] Burningham (John).- Borka Les aventures d'une oie sans plumes, Paris, Seuil, 1963, 1966, 1976, 2002.

[5] Chapouton (Anne-Marie).--Mina la fourmi, Paris, Père Castor Flammarion, 1990, 2000, 2002, 2017.

[6] Clavelet (Magali).- Une place pour Ignace, Saint-Maurice-de Gourdans, Fleur de ville, 2015.

[7] Colard (Bernadette).- Lapinou ne peut pas marcher. Tienne de la petite Bilande (Be), Mols, 1999, 2000, 2010.

[8] De Bodman (Diane), De Zink (Laetitia).-Sacha fait le grand saut : heureux de rebondir face à une difficulté, Bruyère le Châtel, Nouvelle Cité, 2016.

[9] Delval (Marie-Hélène).- Un petit frère pas comme les autres, Montrouge, Bayard, 1991, 1993, 1999, 2003, 2017.

[10] Dunand-Pallaz (Stéphanie), Turrel (Sophie).- Le chat loupé, Lyon, Balivernes, 2015.

[11] Gillieaux (Colette), Barbey (Emilie).-Le pays d'en bas la mer, Talant, Les doigts qui rêvent, 2011, 2012, 2013, 2015.

[12] Goerger (Sonia).- Hugo et Chloé les aînés de la famille sanglier. Les Enfants de la Génétique, 2016.

[13] Goerger (Sonia).- William le petit hippopotame, Les Enfants de la Génétique, 2016.

[14] Georger (Sonia).- Hercule le petit poisson bulle, Les Enfants de la Génétique, 2016.

[15] Guillaumond (Françoise), Oubrerie (Clément).- Poulette crevette, Paris, Magnard, 2001, 2008. 
[16] Hennig (Agathe), Dufresne (Didier).- Chloé ne fait que loucher, Paris, Editons Mango. 2001, 2005.

[17] Karaliitchev (Anguel).- Une larme de maman, Michelbach-le bas, Elitchka. 2014.

[18] Kraus (Robert).-Léo, Paris, L’école des loisirs, 1971, 1972, 1973, 2001.

[19] Pezet (Françoise), Caugant (Catherine).- Ted : une patte ici, trois pattes là-bas, Paris, P'tits Totems, 2009, 2016.

[20] Solotareff (Grégoire), Lecaye (Olga).- Mimi l'oreille, Paris, L’École des Loisirs, $2003,2005$.

[21] Sorin (Céline).-Matachamoua, Paris, L’Ecole des loisirs, 2012.

[22] Stammler (Annie).- Neigeuse la merlette blanche, Paris, L'Harmattan, 1994, 1999, 2000.

[23] Tibi (Marie).- Patrovitt, l'escargot en retard, La Pimpante, Patte de Chevreuil, 2014, 2016.

[24] Tossens (Sophie).- Patouille La petite grenouille dyslexique, Namen (Be), Editions Erasme, 2014.

[25] Wagener (Gerda), Wilkon (Jozef).- Un petit loup si doux, Arles, Actes sud, 2005.

[26] Walcker (Yann), Mercier (Julie).- Léonard est un drôle de canard, Paris, Edition Auzou, 2016.

[27] Weninger (Brigitte), Tharlet (Eve).- Un pour tous, tous pour un, Namur (Be), NordSud, 2006, 2010.

[28] Young (Ed).- 7 souris dans le noir, Toulouse, Éditions Milan,1995, 2001. 
lise.lemoine@univ-rennes2.fr

Ton texte dans le bulletin de psychologie

Chère collègue,

je prépare la copie de ton article pour le Bulletin de psychologie.

J'ai quelques questions à propos de ton texte.

- p. 4, dans lesquelles les animaux donnent à réfléchir sur les dérives dans lesquelles peuvent tomber les sociétés humaines. La ferme des animaux de Georges Orwell (1945), satire de la révolution russe et du stalinisme, est le récit d'une dystopie dans laquelle la révolte : reformuler pour éviter la répétition de dans lesquelles

-p. 7 Tu écris «modestement et de façon très circoncise» c'est plutôt concise ? Ou circonscrite?

-p. 23 en occuper $=$ y occuper $?$

-p. 24 pour appuyer la différence = pour souligner la différence ?

-p. 25 pointe $=$ indique $?$ souligne ?

- p. 26 On peut donc se questionner de l'impact de ces ouvrages clivés sur le handicap sur l'évolution ... = On peut donc se questionner, quant à l'impact de ces ouvrages clivés à propos du handicap, sur l'évolution...?

-p. $26 \mathrm{ou}=$ ou bien OU BIEN = là où = lorsque ?

-p. 27 où $=$ lorsque ?

- Ces références, présentes en bibliographie, ne sont pas citées dans le texte. Je te propose donc de les supprimer (sinon dis-moi où les citer) :

Marcelli (Daniel), Lanchon (Anne). L'enfant, l'animal, une relation pleine de ressources, Foulouse, Erès, 2017.

Reboul (Olivier). Les valeurs de l'éducation, Paris, Presses universitaires de France, 1992.

- Dans le texte, tu cites «Léon (1994)» et en référence on a : Léon (Renée).- La littérature de jeunesse à l'école, Paris, Hachette, 2007.

Attention, merci de me signalez les modifications que tu apportes à ton 
texte en les surlignant en bleu afin que je puisse les repérer et les reporter dans mon fichier. Pense à apporter ces modifications sur le fichier joint à ce courriel.

Merci d'avance pour tes réponses

Stéphane Laurens

\section{bulletin de psychologie}

http://www.bulletindepsychologie.net 